\title{
CARACTERÍSTICAS DE LA COMUNICACIÓN CON ENFERMOS DE CÁNCER EN EL CONTEXTO SANITARIO Y FAMILIAR
}

\author{
CHARACTERISTICS OF THE COMMUNICATION WITH CANCER PATIENTS IN THE \\ HEALTH AND FAMILIAR CONTEXT
}

\author{
Mònica Cunill' y Bernat-Carles Serdà \\ Institut de Recerca i Qualitat de Vida, Universitat de Girona \\ 2 Departament d' infermeria. Universitat de Girona
}

Resumen

Este artículo presenta las características comunicativas que establecen los usuarios, los profesionales sanitarios y los cuidadores que sufren procesos de pérdida. La metodología del estudio corresponde a una triangulación entre métodos en que para el estudio de la misma unidad empírica se utilizan dos estrategias de investigación una cualitativa y otra cuantitativa. El diseño del estudio cualitativo sigue los principios metodológicos de la Grounded Theory. La construcción de la muestra se ha utilizado el muestreo intensivo por representatividad teórica que se concreta a partir de 7 casos ejemplares. El diseño del estudio cuantitativo es descriptivo exploratorio. La muestra corresponde a 63 participantes.

Los resultados muestran los elementos que configuran las características de la comunicación. En este contexto se identifica la existencia de una fractura comunicativa y una contención emocional por parte del profesional que se traslada en el ámbito sociofamiliar aumentando el dolor vivido por el enfermo.

Los indicadores que facilitan la comunicación efectiva en el continuo de la enfermedad corresponden a la importancia del tiempo, la demostración de cuidado, el reconocimiento emocional y la preservación de la esperanza. En el contexto sociofamiliar la dificultad para compartir del dolor se justifica por el miedo a que el otro sufra. Esta protección benevolente incrementa la sensación de dolor que vive el

\section{Abstract}

This paper presents the characteristics of communication that provide users, health professionals and careers who suffer loss processes. The methodology of the study corresponds to a triangulation between methods in which to study the same empirically unit uses two investigation strategies, qualitative and quantitative. The qualitative study design follows the methodological principles of the Grounded Theory. The construction of the sample has been used for intensive sampling theory that represented in 7 cases. The quantitative study design is descriptive and exploratory. The sample corresponds to 63 participants

The results show the characteristics of the communication. In this context, the professional achieve a broken communication and emotional containment. The same result it is identified in the sociofamiliar environment. This situation increases the pain of the patient.

The indicators of an effective communication are the importance of time, the demonstration of care, emotional recognition and preservation of hope. In the sociofamiliar context the difficulty to share the emotion it is justified by the fear that the family or friend suffers. This benevolent protection increases the sensation of pain which the patient lives in silence and solitude.

In conclusion, the promotion of collaborative communication and emotional self-regulation

\section{Correspondencia:}

Mònica Cunill Olivas. Universidad de Girona.

Departament de Psicologia. Plaça Sant Domènec, 3. 17071. Girona. monica.cunill@udg.edu Bernat-Carles Serdà.

Universidad de Girona. Departament d'infermeria. Emili Grahit, 77. 17071. Girona. bernat.serda@udg.edu 
paciente en silencio y en soledad.

Como conclusión, la promoción de una comunicación colaborativa y afectiva facilita la autorregulación emocional del paciente y media la reconceptualización de la calidad de vida tanto del afectado como de la red de apoyo.

Palabras clave: Proceso de pérdida, comunicación, cáncer, calidad de vida. facilitates the patient's emotional and mediates the re-conceptualization of quality of life of individuals as network support.

Key words: Loss process, communication, cancer, quality of life.

\section{INTRODUCCIÓN}

Vivir un proceso de pérdida, ya sea como consecuencia de sufrir una enfermedad grave o por la muerte de un ser querido, es una de las situaciones de mayor impacto para la persona y su entorno. Desde un modelo holístico de la salud, este impacto afecta a la totalidad de la persona (a nivel físico, emocional relacional, intelectual y espiritual) y repercute en el sistema familiar ${ }^{(1)}$.

Todo proceso de pérdida genera estados de malestar psicológico y requiere de la puesta en marcha de mecanismos de adaptación a las nuevas circunstancias ${ }^{(2)}$. Los datos epidemiológicos muestran que son muchas las personas que desarrollan algún tipo de dificultades a lo largo del proceso y que alrededor del 30\% necesitan de ayuda profesional(3). Uno de los principales factores que influyen en el ajuste de la persona es el manejo adecuado de la información y de la comunicación por parte de todos los implicados en el proceso (paciente, profesionales y familia) y en los diferentes contextos (sociosanitario y familiar).

La comunicación efectiva integra un componente informativo y otro psicoemocional $^{(4)}$. En el contexto sociosanitario la comunicación efectiva requiere la transmisión de un contenido relativo al diagnóstico y al pronóstico de la enfermedad por parte del profesional, y a un alto nivel de complicidad interpersonal ${ }^{(5)}$. Esta complicidad se genera a partir de la creación de un espacio de confianza y seguridad donde existe una relación de interdependencia entre el profesional y la persona afectada, en el sentido de que el profesional es experto en las técnicas y el paciente en identificar sus necesidades ${ }^{(6)}$. Además, este espacio permite a la persona compartir sus dudas, deseos y preocupaciones y expresar de manera libre sus emociones.

En la actualidad, el sistema sanitario consigue una gran efectividad en la intervención física y funcional predominando un estilo comunicativo dirigido ${ }^{(7)}$. Los estudios describen un déficit en la comunicación colaborativa entre equipo asistencial, enfermos y cuidadores (amigos, familia). Este recurso comunicativo es necesario para afrontar la adaptación a la enfermedad y el proceso de pérdida ${ }^{(8)}$.

El objetivo del estilo colaborativo es elaborar un plan de cuidados de forma compartida. Este estilo se fundamenta en centrar la atención en las necesidades del paciente a partir de la actitud de aceptación incondicional, autenticidad, empatía, respeto, compasión y afecto ${ }^{(6)}$. Esta comunicación requiere disponer de una atmósfera adecuada y un tiempo para atender la emoción y resolver dudas. Así mismo, implica una capacidad de escucha y de indagación para explorar intencionalmente la situación decidiendo de forma compartida los recursos para el afrontamiento ${ }^{(9-11)}$. Los profesionales que practican este estilo comunicativo utilizan una información ajustada, reconocible y significativa, calibrando el umbral comunicativo del afectado. 
Los efectos de este tipo de intervención proporcionan un sentimiento de esperanza y una capacidad para utilizar, de forma autónoma, los recursos necesarios para afrontar el malestar y la incertidumbre que conlleva el proceso de pérdida, mejorando la calidad de vida y de muerte.

La comunicación dirigida se caracteriza por priorizar la atención en las necesidades del profesional a partir de los aspectos técnicos sin considerar adecuadamente la dimensión psicoemocional. Así pues, el estilo comunicativo resultante es fracturado, inconsistente y borroso (con falta de claridad). El efecto de este estilo comunicativo, puede generar un cuadro emocional caracterizado por insatisfacción, distrés, desesperanza, miedo, frustración y depresión ${ }^{(12)}$.

Los factores que explican el déficit en el campo de la comunicación psicoemocional corresponden a una falta de formación del profesional sanitario en el manejo de situaciones complejas, a la presión asistencial del profesional, al nulo reconocimiento curricular de las actitudes, al miedo y a la falta de reconocimiento del trabajo sobre la muerte como necesidad básica de la persona enferma ${ }^{(13)}$.

El contexto sociofamiliar es otro de los pilares fundamentales en la evolución de la adaptación del proceso de pérdida. La evidencia empírica confirma que durante el proceso de pérdida y duelo los afectados experimentan un proceso de cambio y transformación a nivel multidimensional, (espiritual, valores, físico, social, psicológico), que les capacita a la adaptación progresiva y autorregularse al cambio. Este es el motivo de la discrepancia observada entre el estado vivido y la elevada Calidad de Vida registrada(14,15). Al igual que en el sistema sanitario en la red natural se observan dos tipos de acompañamiento: El afectivo y el evitativo. El primero se caracteriza por la existencia de canales de comunicación abiertos y un clima natural y afectivo en el que se puede compartir e intercambiar información, así como, expresar emociones en el interior de la familia $^{(16)}$. En esta misma línea, Kubler-Ross señala la importancia para la persona del apoyo y de la compañía, así como de la escucha oportuna de sus sentimientos ${ }^{(17)}$. Los efectos de este estilo comunicativo permiten integrar las necesidades de la persona enferma conjuntamente con las de los familiares, iniciando un proceso de afrontamiento compartido. En esta línea es más probable que enfermo y familia integren los nuevos roles y encuentren un significado de pertenencia y apoyo familiar en el proceso de pérdida ${ }^{(18)}$.

Por otro lado, la familia que presenta un estilo evitativo muestra dificultades para expresar preocupaciones y emociones entre las personas directamente vinculadas. La tendencia a la sobreprotección se manifiesta con la negativa a compartir información o con actitudes de negación del proceso de pérdida que dificultan el proceso de afrontamiento del afectado y de sus seres queridos. Otro factor presente es la incapacidad de los familiares para gestionar sus propias emociones y poder ofrecer un apoyo eficaz ${ }^{(19)}$. Habitualmente la red natural presenta un grado elevado de afectación emocional o sufrimiento. En estos casos, el sistema sanitario debería integrarlos en su plan de cuidados.

La comunicación adecuada y oportuna es un factor protector en situaciones de pérdida y duelo ya que permite la obtención de recursos efectivos para la ayuda y el apoyo ${ }^{(20,21)}$. Así pues, a pesar que la comunicación es una de las herramientas básicas en procesos de duelo, la experiencia clínica indica que en situaciones de sufrimiento la comunicación resulta especialmente compleja. En algunos casos, se construye un muro de silencio alrededor de la enfermedad y de la muerte que dificulta la adaptación y la correcta elaboración del duelo ${ }^{(1,22)}$. Este trabajo incluye dos 
estudios, uno utiliza metodología cualitativa y el otro metodología cuantitativa, con el objetivo de describir las características de la comunicación entre el enfermo de cáncer y la red formal (profesionales sanitarios) y éste y su red de apoyo natural (pareja, familia y amigos) en el contexto sociosanitario y familiar.

\section{Estudio 1.- Características de la comunicación en el sistema sanitario}

Método

El diseño del estudio 1 es de tipo cualitativo. Sigue los principios metodológicos de la Grounded Theory y corresponde a un estudio de $\operatorname{casos}^{(23)}$. Esta propuesta metodológica permite superar el estudio de la comunicación como un índice de resultado y permite identificar los factores de proceso que influencian el proceso de comunicación eficaz.

\section{Muestra}

Para la construcción de la muestra se ha utilizado el muestreo intensivo por representatividad teórica ${ }^{(24)}$. En este contexto se han escogido 35 participantes y se han agrupado en 7 casos ejemplares, 4 per- tenecientes a enfermos de cáncer y 3 a los cuidadores. Los casos relativos a los enfermos de cáncer se concretan a partir del género, la edad, el tipo de cáncer, el tipo de tratamiento, el nivel sociocultural, el estado civil y el tiempo de experiencia con el sistema desde el inicio del tratamiento paliativo. Por lo que se refiere a los cuidadores esto se definen a partir de la edad, el tipo de cáncer y el estadio de la enfermedad (ver Tabla 1).

\section{Procedimiento}

La información se ha generado a partir de la siguiente entrevista semiestructurada:

- ¿Cómo se encuentra?

- ¿Cómo describiría su relación con el profesional?

- ¿Quién le ha ayudado /quién le ha escuchado en sus dudas, miedos, temores de la enfermedad y tratamiento/proceso de pérdida?

- ¿Qué le ayuda a establecer o mantener una comunicación eficaz?

- ¿A quién recurre sobre sus dudas, interrogantes que genera la enferme$\mathrm{dad} /$ proceso de pérdida?

- ¿Qué piensa sobre el servicio sociosanitario? (necesidades cubiertas/sin cubrir)

Tabla 1. Descripción de la muestra del estudio 1

\begin{tabular}{|llllllll|}
\hline Caso & 1 & 2 & 3 & 4 & 5 & 6 & 7 \\
\hline Edad & $65-70$ & $45-65$ & $55-65$ & $65-70$ & $35-45$ & $75-85$ & $35-45$ \\
Género & Mujer & Hombre & Mujer & Hombre & Mujer & Familiar & Familiar \\
\hline $\begin{array}{l}\text { Tipo de cáncer } \\
\text { Mama }\end{array}$ & Próstata & Mama & Próstata & Mama & Próstata & Mama \\
$\begin{array}{l}\text { Experiencia } \\
\text { con el sistema }\end{array}$ & 2001 & 2001 & 2007 & 2004 & 2004 & 2001 & 2007 \\
$\begin{array}{l}\text { Tipo } \\
\text { tratamiento }\end{array}$ & Quim & Q & H & C & R & Q & H \\
\hline
\end{tabular}

Q: quirúrgico; R: Radioterapia; Quim: Quimioterapia; H: hormonal; C: combinados. 
Todas las entrevistas fueron grabadas con un magnetófono, con el consentimiento previo de los participantes.

El procedimiento analítico de la información corresponde a la generación de categorías conceptuales aplicando el Método de Comparaciones Constantes (MCC) de la Grounded Theory ${ }^{(25)}$. Además se realiza la triangulación entre informantes y analistas con el objetivo de garantizar la validación de los resultados cualitativos.

\section{Resultados}

Los resultados muestran los elementos que configuran las características de la comunicación y se estructura en tres ejes temáticos: Características comunicativas del profesional, características comunicativas del paciente y los indicadores de la comunicación efectiva.

Las características comunicativas del profesional.

\section{La fractura comunicativa}

En este contexto los pacientes sienten cubierta la dimensión física y funcional en los estadios iniciales de la enfermedad con el tratamiento curativo, pero se identifica como deficitaria y borrosa en el periodo de cambio de la terapia curativa hacia la etapa paliativa (interperiodo). Los informantes describen la falta de atención comunicativa efectiva que garantice la atención de calidad. Caracterizan la comunicación como fragmentada y dirigida. Esta barrera comunicativa delimitada por el profesional es muy difícil traspasar.

"...A la primera visita vale, pero ya a la segunda y al no contestar ninguna de mis preguntas de cómo iba el tratamiento, presentí que las cosas no iban bien, sufría mucho, tuve varios ataques de angustia en casa, sola..." (Caso 5).
"Cuando tuvo la metástasis no le atendían como necesitaba, lo pasó muy mal, se sintió abandonada" (caso 7).

\section{La contención emocional}

La comunicación psicoemocional se encuentra desatendida a lo largo de todo el proceso. De forma general, la comunicación emocional incomoda al profesional. Los pacientes identifican un proceso de filtraje de la información que acaba focalizándose o desviándose hacía los aspectos técnicos y acaba siendo un contacto emocionalmente neutro. Esta práctica es vivida como poco útil ya que no tiene la capacidad de manejar la información prioritaria del afectado y de su red de cuidadores. El profesional sanitario es incapaz de reconocer las emociones y en consecuencia evita o no aborda ni interviene de forma empática a la demanda. Ante la imposibilidad de afrontar la comunicación emocional del afectado el profesional se remite al concepto o elemento técnico que causa una emoción determinada más que a la emoción en si misma. Habitualmente el profesional bloquea la emoción distanciándose de ella y así poder evitar una situación que perciben como potencialmente estresante. Es poco probable que el profesional sanitario ofrezca la respuesta afectiva de apoyo emocional para mejorar la fortaleza natural del paciente y afrontar el distrés secundario (19). El resultado de la interacción psicoemocional usuarioprofesional es de vacío, descontento, frustración y desesperanza. El efecto de esta interacción comunicativa dificulta la regulación emocional del paciente.

"El Dr X mira el PSA y me pregunta cómo me encuentro, cuando le explico que me siento solo, me mira y dice que esto el no lo puede arreglar..." (Caso 4). 
Una respuesta efectiva por parte del profesional supone la continuidad de la comunicación de la emoción de una forma legítima y natural con una alta posibilidad de resolverla.

"Cuándo me programaba el tratamiento (médico) rompí a llorar, me preguntó que le ocurre $X$ ? Le conté que el miedo no me dejaba dormir... que tenía miedo a todo a que mis niños se quedaran solos, miedo a no volverlos a ver... Este día se dio cuenta de la gravedad del problema y que debíamos buscar ayuda..." (Caso 3).

En cambio, la comunicación no efectiva supone la interrupción en la expresión de la emoción, quedando sin resolver y dejando al paciente con la necesidad de retomarla.

"El día de la consulta le conté que estaba deprimida, hizo un silencio largo y continuó hablando de otro tema como si no hubiera dicho nada" (Caso 1).

"Le pregunté si mi marido estaba en una fase grave y si tenía alguna posibilidad de curarse, me dijo: ahora no piense en esto no le soluciona nada" (Caso 6).

Los usuarios expresan que la comunicación psicoemocional en su conjunto se atiende de forma parcial y limitada. La expresión habitualmente más atendida por el profesional es la tristeza ya que despierta una identificación primaria de necesidad de protección y por ende se muestra receptivo y empático. La expresión del miedo es consecuencia de la incertidumbre del proceso de progresión y recurrencia de la enfermedad, así como, las posibilidades de conseguir una oportunidad de tratamiento curativo. Así pues, el profesional sanitario atiende con mayor calidad la tristeza y, posteriormente el miedo y la angustia.

"El primer día de quimio me entró un ataque de angustia: Todo el rato repetía entre sollozos, tengo miedo, no quiero que mis hijos se queden solos. La enfermera no supo reaccionar ni qué decir, cada vez que la miraba desviaba la mirada. La siguiente quimio estaba más calmada y muy triste, entonces me dijo: "no se preocupe señora todo irá bien" (Caso 5).

Cuando el paciente siente que se agotan sus recursos personales para afrontar el impacto emocional aumenta el riesgo de dejarse arrastrar por la emocionalidad negativa y se experimenta una sensación total de pérdida del control. Esta situación mantenida implica un alto grado de sufrimiento en el afectado difícil de sostener y a largo plazo puede derivar en enfermedades físicas o mentales. Es en este periodo cuando debe plantearse la necesidad de ayuda profesional.

"... Cuando ves tantas cosas encima y que te sientes mal y sólo, no sabes cómo lo vas a hacer, te hundes... siempre pienso que esta tristeza me va a reproducir el cáncer en otro lado del cuerpo" (Caso 2).

Las características comunicativas del paciente

Si nos centramos en la red de apoyo natural, los usuarios también presentan una dificultad comunicativa, de manera que aplican una técnica de filtraje deliberado y controlado con las personas significativas en los distintos contextos sociales. La manera de gestionar la emoción en este entorno consiste en trivializar e invisibilizar su estado emocional o bien utilizar 
máscaras y conductas protectoras en el entorno habitual.

"Los problemas de mi enfermedad sólo los puedo contar a mi madre. En el trabajo serían compasivos y esto no ayuda. Mi marido es demasiado sensible y protector. Los niños son demasiado pequeños para entenderlo,...no quiero que sufran para nada" (Caso 5).

La fractura comunicativa retroalimenta negativamente la sensación de soledad y aislamiento emocional en el contexto sociofamiliar del afectado. Estos factores justifican que en la mayoría de los casos el cuadro emocional no queda resuelto. En este punto, es cuando se plantean iniciar un plan de autocuidado emocional o pedir ayuda del profesional experto.

"...Cuándo estoy en casa y pienso $X$ aguanta, no te vayas abajo...cuando los niños me preguntan si me he curado me cierro en el lavabo y rompo a Ilorar... no lo puedo parar. Necesito pedir ayuda o ir al psicólogo" (Caso 5).

\section{Indicadores de la comunicación efectiva}

Las percepciones de los enfermos en relación a los factores mediadores de la comunicación efectiva corresponden a: la importancia del tiempo, la demostración de cuidado; el reconocimiento emocional y la preservación de la esperanza.

\section{La importancia en la calidad del tiempo}

El sistema sociosanitario no destina el tiempo suficiente para conseguir la calidad en los cuidados. Los usuarios valoran muy positivamente la capacidad del profesional para conseguir tiempo de dedicación hacia los aspectos informativos importantes del usuario. Aunque más que los minutos invertidos se valora especialmente la ca- lidad y la forma de distribuir y dedicar el tiempo fijado de visita.

"Es un gran profesional. Aunque sólo entrar a la consulta me espera con los resultados de los análisis y ni tan solo me pregunta, ¿cómo se encuentra"? (Caso 1).

Los indicadores de calidad asistencial identificados por el usuario se basan en unos factores clave: el contacto visual directo, la inclusión de una técnica de escucha activa, la generación de un espacio comunicativo cómodo y sin interrupciones y el lenguaje no verbal receptivo y abierto. El periodo en que el usuario detecta una menor calidad asistencial corresponde a la fase de cambio de tratamiento: de curativo a paliativo. En estos casos, la falta de dedicación de tiempo del profesional lo percibe como el desinterés (abandono) del profesional para buscar una opción de cura para su enfermedad.

"Desde que me dio las hormonas ya no me dedica tiempo, y no me programa visitas. Me llama la secretaria y me dice que continúe igual" (Caso 4).

La demostración de cuidado.

Los usuarios trazan perfectamente la línea divisoria entre los aspectos de cuidado que pertenecen a la conducta del profesional de los que pertenecen a su entorno de red social. El problema emerge cuando los aspectos comunicativos y de cuidados no se producen ni se resuelven en el entorno sociosanitario, entonces se genera una situación de emergencia para conseguir solucionar las dudas y necesidades de aclaración. Los informantes confirman que al no resolver aspectos clave de la comunicación en el entorno estructurado deben dirigirse a la red social de cuidados. La respuesta técnica al no provenir 
de la figura del profesional genera desconfianza. Mientras que el apoyo psicoemocional en el plan de cuidados de la red natural resulta totalmente eficaz.

"Estaba desconcertada, no sabía si mi enfermedad era grave tuve que clarificar mi diagnóstico con un amigo médiCo" (Caso 1).

En relación a los cuidados, los usuarios describen al profesional ideal como: una figura formada, conocedora del historial de la enfermedad, asertiva y empática, con capacidad para atender los aspectos emocionales y para facilitar los recursos adecuados para el afrontamiento y colaborativa para elaborar un plan de cuidados compartido en los diferentes estadios de la enfermedad.

"Cuando supe que tenía metástasis dejé de luchar, me abandoné. La enfermera me dijo que nunca se sabe cuánto podría estar así. Que debía seguir una dieta, practicar ejercicio, técnicas de relajación para controlar su evolución: esto podía hacerlo por mi misma, tenía que sentir que podía hacer algo contra este mal" (Caso 3).

\section{El reconocimiento emocional}

Una estrategia de ayuda es el reconocimiento emocional del miedo durante la comunicación con el profesional. El miedo se identifica en la fase del diagnóstico terminal o al fracaso del tratamiento y deriva del efecto de pérdida. Habitualmente el miedo es consecuencia del sufrimiento que conlleva la enfermedad, a la pérdida de las facultades y a la dignidad, más que a la propia muerte. Corresponde a la incertidumbre a lo que tiene que acontecer "miedo a lo que va a venir". El miedo se confirma e intensifica con el sentimiento de abandonado por el sistema sanitario y el sistema de apoyo social. En estos casos una planificación de cuidados incluyendo y respetando las voluntades anticipadas de la persona enferma, minimiza enormemente el síntoma de miedo.

"Me siento débil y enferma, me da miedo sufrir. Esto no me deja continuar" (Caso 1).

Los usuarios requieren una capacidad de escucha del miedo asociado a la imposibilidad de luchar contra la enfermedad. No necesitan una postura paternalista ni actitudes compasivas en el plan de cuidado; tan solo aceptar este espacio psicológico en el que se pueda expresar el miedo como un aspecto natural que emerge durante el proceso de pérdida.

"Cuándo conté mis miedos a mis amigas pude continuar" (Caso 1).

\section{La preservación de la esperanza}

El usuario identifica la esperanza como una condición inherente en la comunicación efectiva en el continuo de la enfermedad. La esperanza es un valor comunicativo necesario para mantener la interacción interpersonal y superar el proceso de pérdida que conlleva la enfermedad.

"Después de pasar tantas noches sin dormir cuando el Dr me dijo: buscaremos la mejor forma para calmar este dolor, volví a coger esperanza y pensé que aún no era hora de rendirme..." (Caso 4).

Los informantes describen un continuo de estados emocionales progresivos vinculados al estado o situación de la enfermedad en el que la percepción de muerte es ubicua (omnipresente). Este continuo corresponde al inicio con un estado de negación en la que describen un cuadro de 
miedo, ansiedad de un futuro incierto, con sentimientos de impotencia e indefensión. La siguiente fase continua con el enojo y la rabia, posteriormente la negociación y lucha, continuando con la depresión y el desespero, hasta llegar a la aceptación de la pérdida con la vivencia de un encadenaje de continuos fracasos y finalmente el estado de esperanza. En este contexto la esperanza se arraiga en este estado de la vida con el objetivo de poder cumplir con los asuntos pendientes. La esperanza va ligada a la incertidumbre del pronóstico del tratamiento. Esperanza a la curación, a vivir con la máxima normalidad y calidad posible, al acompañamiento presente de la pareja y/o familiar. La reconciliación con el confort entre la vida y la muerte. A pesar de todo, la esperanza tiene una connotación positiva en la que se describen estados de vida y aspectos positivos vinculados con el bienestar de él/ella y su red. En este entorno se valora más el ser y el estar que el tener y hacer. A partir de la red natural se genera un nuevo periodo especial de vida de fuerza compartida en el que también se rompen con otros lazos antiguos.

"Ahora quiero vivir con mi gente, haciendo las cosas que me apetecen y me hacen feliz. Se acabó perder el tiempo con tonterías" (Caso 4).

"Cuando mamá estuvo enferma cada día nos reuníamos todos los hermanos en casa. Siempre estuvo acompañada, estuvimos luchando hasta el final. (Caso 7).

\section{Estudio 2.- Características de la comunicación en el contexto familiar}

Método

El presente estudio descriptivo, se basa en un diseño no experimental transversal.
Muestra

La muestra está formada por 63 personas que sufren un proceso de pérdida, de las cuales un $55,6 \%$ son pacientes oncológicos, un $41,3 \%$ han perdido a un ser querido y un 3,2\% padecen ambas situaciones. Los participantes tienen una edad media de 48,2 años $(d t=12,3)$ y el $85 \%$ son mujeres.

\section{Procedimiento}

El procedimiento de recogida de información se realiza a partir de un breve cuestionario de elaboración propia. Además de los datos demográficos, el cuestionario incluye las siguientes preguntas:

1. ¿En qué medida puede hablar de lo que le preocupa con su pareja, con algún familiar y con algún amigo?

2. ¿En qué medida puede expresar sus emociones con su pareja, con algún familiar y con algún amigo?

3. ¿En qué medida se siente comprendido/a por su pareja, por algún familiar y por algún amigo?

4. Lo que hace que me sea difícil hablar de mis preocupaciones, compartir mis emociones con mi pareja, con algún familiar y con algún amigo es:

a. tener poca confianza.

b. sentir que no me comprende.

c. poder preocuparla.

d. pensar que al compartir hago daño.

e. que me cuesta expresarme.

f. que soy así.

g. que me siento débil.

h. y pensar que él o ella no podrá soportarlo.

Se utilizó una escala tipo likert, donde 0 indicaba "En absoluto" y 10 "Muchísimo". Los resultados han sido analizados con el paquete estadístico SPSS v15. 


\section{Resultados}

Por lo que se refiere a compartir las preocupaciones relacionadas con su estado, encontramos que los pacientes las expresan de manera moderada, sin existir diferencias significativas en función del vínculo ni del sexo. La media es de 6,29 $(d t=3,23)$ con la pareja, de 5,69 $(d t=3,43)$ con algún familiar y de $6,29(\mathrm{dt}=3,44)$ con algún amigo (ver figura 1).

El principal motivo por el que a los participantes les resulta difícil compartir aquello que les preocupa tanto con la pareja, como con algún familiar o amigo es el hecho de pensar que "puedo preocuparla" y en segundo lugar "pienso que al compartir mi preocupación le hago daño" (ver tabla 2).

En relación a compartir la expresión de diferentes emociones con el entorno inmediato encontramos que también es moderada. Así mismo, los resultados nos indican que éstas se expresan en mayor medida con algún amigo (media $=5,92$ $\mathrm{dt}=3,64$ ) que con la pareja (media $=4,81$ $\mathrm{dt}=3,71)$ siendo esta diferencia estadísticamente significativa ( $t=-2,04 ; \mathrm{P}=0,04)$.
No encontramos diferencias significativas en el caso de la expresión emocional con la familia (media $=5,10 \mathrm{dt}=3,45$ ) en comparación con la pareja $(t=-0,363 ; p=0,72)$ o con algún amigo $(t=-1,44 ; p=0,17)$ (ver figura 2). Existen diferencias significativas en función del sexo en el caso de compartir las emociones con la pareja ( $\mathrm{X} 2=4,27$; $\mathrm{p}=0,046$ ), en el sentido de que son los hombres quienes expresan más.

Por lo que se refiere a las principales razones que dificultan la expresión emocional, éstas, al igual que sucedía con la preocupación hace referencia a la posibilidad de preocupar y de hacer daño a la pareja o a algún familiar. En el caso de los amigos, el motivo que destaca es el pensar que existe falta de comprensión (ver tabla 3).

Finalmente se analizan los resultados referentes a sentirse comprendido por las personas cercanas. Los participantes se sienten bastante comprendidos por su pareja (media $=6,96 ; d t=3,52$ ), por los familiares (media $=7,21 ; \mathrm{dt}=3,41)$ y por los amigos (media $=7,21 ; \mathrm{dt}=3,52$ ) (ver figura 3) sin existir diferencias significativas en función del vínculo ni del sexo.

Figura 1. Media de la variable compartir las preocupaciones en función del vínculo

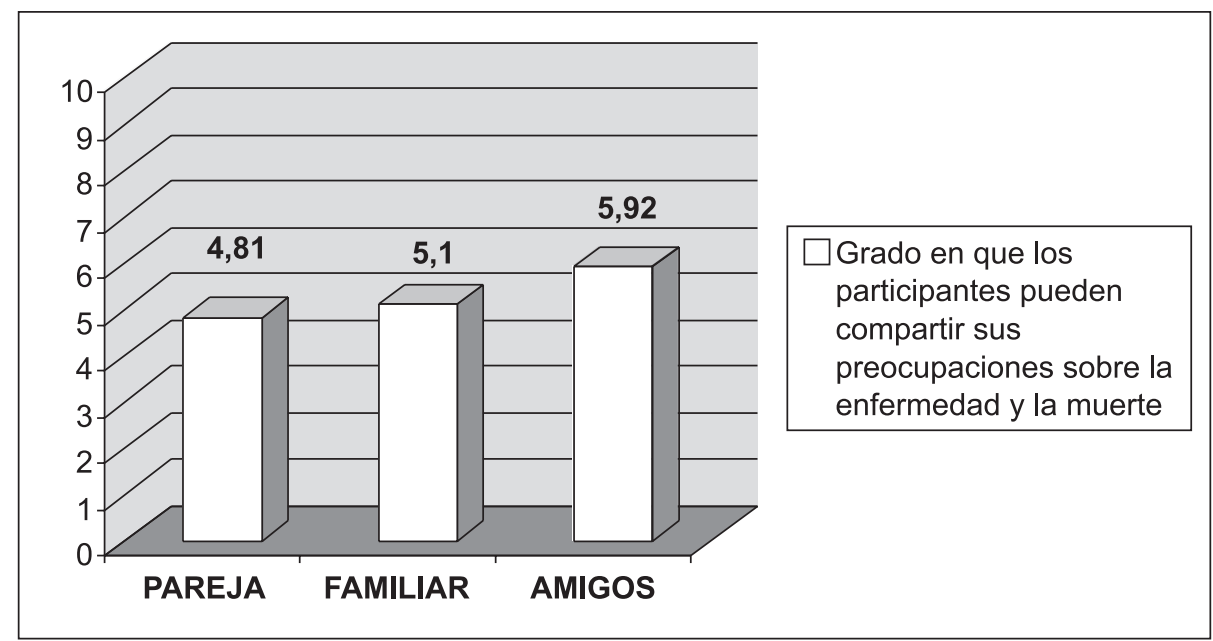


Tabla 2. Medias y desviaciones típicas de los motivos por los cuales es difícil compartir las preocupaciones en función del vínculo

\begin{tabular}{|c|c|c|c|}
\hline & $\begin{array}{l}\text { PAREJA } \\
\text { Media (dt) }\end{array}$ & $\begin{array}{l}\text { FAMILIAR } \\
\text { Media (dt) }\end{array}$ & $\begin{array}{l}\text { AMIGO } \\
\text { Media (dt) }\end{array}$ \\
\hline Tengo poca confianza. & $3,03(3,5)$ & $3,61(3,7)$ & $2,94(3,35)$ \\
\hline $\begin{array}{l}\text { Siento que no me } \\
\text { comprende. }\end{array}$ & $2,41(2,87)$ & $3,22(3,58)$ & $3,74(3,54)$ \\
\hline Puedo preocuparla. & $6,04(3,74)$ & $6,74(3,3)$ & $4,50(3,29)$ \\
\hline $\begin{array}{l}\text { Pienso que al compartir mi } \\
\text { preocupación le hago daño. }\end{array}$ & $6,00(4,06)$ & $6,54(3,35)$ & $3,50(2,98)$ \\
\hline $\begin{array}{l}\text { Me cuesta expresar mi } \\
\text { preocupación con todo el } \\
\text { mundo. Soy así. }\end{array}$ & $3,97(3,42)$ & $4,63(3,71)$ & $4,20(3,71)$ \\
\hline Me siento débil. & $4,17(3,78)$ & $3,62(3,58)$ & $3,51(3,64)$ \\
\hline $\begin{array}{l}\text { Pienso que él /ella no podrá } \\
\text { soportarlo }\end{array}$ & $4,21(3,33)$ & $4,68(3,69)$ & $2,65(2,82)$ \\
\hline
\end{tabular}

Figura 2. Media de la variable compartir las emociones en función vínculo

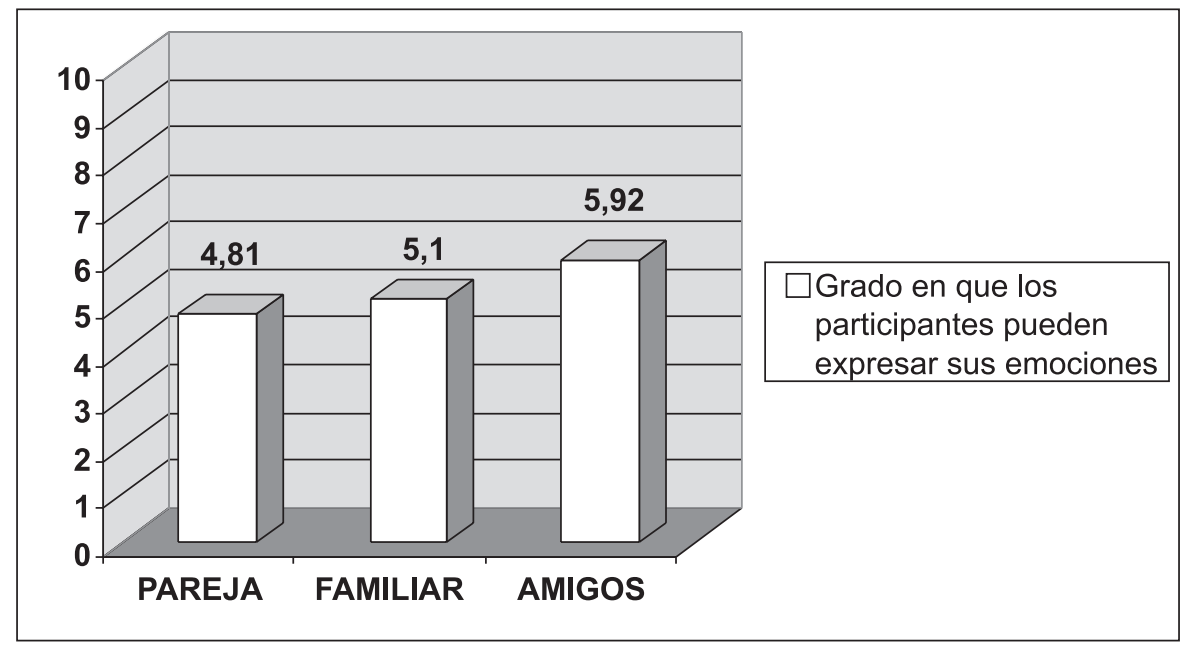


Tabla 3. Medias y desviaciones típicas de los motivos por los cuales es difícil
compartir las emociones en función del vínculo

\begin{tabular}{|c|c|c|c|}
\hline & $\begin{array}{l}\text { PAREJA } \\
\text { Media (dt) }\end{array}$ & $\begin{array}{l}\text { FAMILIAR } \\
\text { Media (dt) }\end{array}$ & $\begin{array}{l}\text { AMIGO } \\
\text { Media }(d t)\end{array}$ \\
\hline Tengo poca confianza. & $2,16(3,38)$ & $3,06(3,33)$ & $3,39(3,74)$ \\
\hline $\begin{array}{l}\text { Siento que no me } \\
\text { comprende. }\end{array}$ & $3,16(3,71)$ & $2,71(3,06)$ & $4,20(3,84)$ \\
\hline Puedo preocuparla. & $6,13(3,58)$ & $6,48(3,42)$ & $3,65(3,26)$ \\
\hline $\begin{array}{l}\text { Pienso que al compartir mis } \\
\text { emociones le hago daño. }\end{array}$ & $6,17(3,45)$ & $6,97(3,03)$ & $3,81(3,13)$ \\
\hline $\begin{array}{l}\text { Me cuesta expresar mis } \\
\text { emociones con todo el } \\
\text { mundo. Soy así. }\end{array}$ & $3,87(3,46)$ & $4,19(3,47)$ & $4,10(3,68)$ \\
\hline Me siento débil. & $3,23(3,25)$ & $3,75(3,57)$ & $3,90(3,34)$ \\
\hline $\begin{array}{l}\text { Pienso que él /ella no podrá } \\
\text { soportarlo }\end{array}$ & $3,90(3,14)$ & $4,19(3,30)$ & $2,93(2,93)$ \\
\hline
\end{tabular}

\section{Discusión y conclusiones}

Los resultados de esta investigación confirman la existencia de una fractura comunicativa en los procesos de enfermedad grave en el sistema sanitario. Esta aportación va en la línea en lo expuesto por Navarro ${ }^{(6)}$. Esta dificultad comunicativa se traslada al contexto sociofamiliar a partir de la no expresión de la emoción por parte del afectado.

A partir del estudio cualitativo se observa que el profesional es incapaz de cubrir la demanda psicoemocional de la persona afectada. Coincidiendo con Morita y Lucille la intervención del profesional se focaliza en los aspectos técnicos y desatiende las emociones naturales que surgen a lo largo del proceso ${ }^{(26,27)}$. El efecto de este estilo comunicativo dificulta la autorregulación emocional del paciente. Para facilitar la adaptación a la nueva situación, el profesional debería realizar una primera acogida emocional y con una toma de decisiones compartida identificar los recursos útiles para que ambos sean capaces de gestionar la situación.

De forma general el profesional se siente capacitado para atender emociones de tristeza, abatimiento, desamparo y depresión. En cambio se observa una falta de habilidad para gestionar la expresión de rabia, frustración y angustia(28).

Los motivos que justifican esta desatención emocional en el contexto sanitario se relacionan con la falta de habilidades y herramientas del profesional para establecer una relación de ayuda integral durante el proceso de enfermedad. En el momento en que la enfermedad entra en fase paliativa se incrementa el descontento con la atención recibida en el sistema sanitario. Esto podría relacionarse con el hecho de que el afectado y su familia se sienten abandonados por el sistema y con la falta de recursos del profesional que requiere de habilidades 
Figura 3. Media de la variable sentirse comprendido en función del vínculo

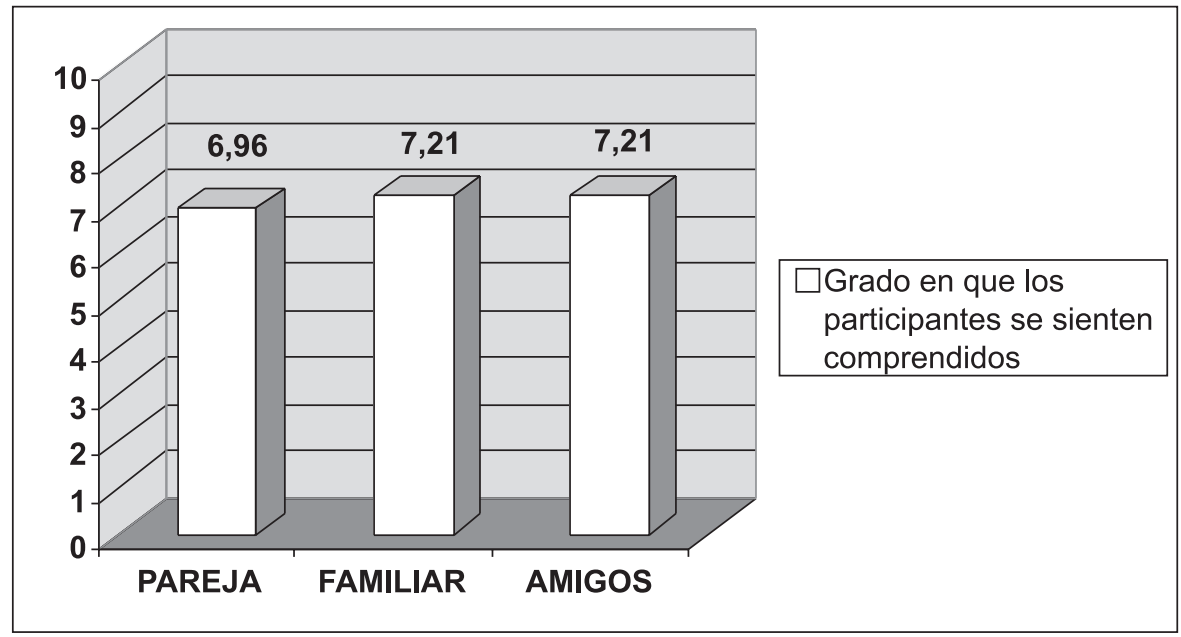

más humanas y menos técnicas para acompañar en este momento de sufrimiento.

Ambos estudios coinciden en que la contención emocional continúa en el contexto sociofamiliar. En este contexto, el principal motivo de la dificultad para compartir el dolor es el miedo a que el otro sufra. Seguramente esta decisión de no compartir la vivencia de la pérdida está basada en una decisión de "protección benevolente", es decir, se intenta proteger a los seres queridos del dolor, pero paradójicamente, se consigue el efecto contrario: se incrementa el dolor ya que éste es vivido en silencio y en soledad.

Este nuevo estado se caracteriza por un sesgo intencional en el intercambio comunicativo que se retroalimenta de manera negativa y genera una desconexión de la dimensión emocional: el usuario no expresa, la familia no pregunta y el profesional relega. Esta desatención emocional a tres bandas mantenida en el tiempo genera un elevado nivel de sufrimiento y una alta comorbilidad añadida.

La calidad de vida del usuario se ve afectada negativamente y se ve obligado a vivir el proceso en soledad y aislamien- to al no poder integrar, naturalizar y legitimar el nuevo estado en su vida. Así mismo, los familiares son copartícipes al no afrontar la situación de forma explícita y no integrar la enfermedad en el entorno familiar. Este es el momento en que la adaptación al proceso de pérdida depende exclusivamente de los recursos personales del afectado, para afrontar la situación o solicitar ayuda a otro tipo de profesional.

De acuerdo con el estudio de Eliott y Olver (2009) ${ }^{(29)}$, la esperanza y el optimismo son decisivos para conseguir que la persona afectada se autorregule y se adapte en el continuo de la enfermedad, cuando estos aspectos fracasan, supone que se produzca el efecto de pérdida de los objetivos de afrontamiento de la enfermedad con el riesgo que conlleva de abandono.

De acuerdo con Munday (2009)(30) la persona que sufre un proceso de pérdida debe tener la oportunidad de decidir cómo vivir esta situación. Esta libertad de elección sólo es posible si dispone de la información adecuada y si sus necesidades psicológicas, emocionales y existenciales 
han sido atendidas por los profesionales y por su entorno familiar, desde el respecto de su propio bioritmo ${ }^{(31)}$.

Los resultados de este estudio ponen de manifiesto la necesidad de integrar la atención de la dimensión emocional desde el principio de la intervención o intercambio comunicativo.

La intervención asistencial debería incluir las habilidades de comunicación entre las competencias del profesional sanitario con el objetivo de superar esta visión fragmentaria. El espacio comunicativo debe incluir las emociones, las vivencias, los valores y la propia historia de vida, observando a la persona durante el proceso de pérdida desde una perspectiva holística. El proceso de pérdida supone un proceso de reconceptualización de la calidad de vida. En este marco aspectos que suponían una elevada calidad de vida en el estado actual no son decisivas mientras que se priorizan otras dimensiones que anteriormente al proceso de pérdida no resultaban significativas.

\section{REFERENCIAS BIBLIOGRÁFICAS}

1. Rodríguez $\mathrm{A}$, Ruiz $\mathrm{Y}$, Recrespo $\mathrm{M}$. Intervención familiar para el manejo psicológico en pacientes oncológicos con mal pronóstico en el hospital de la Misericordia. Rev Col Psicol 2004;(13):90101.

2. Neimeyer R. Aprender de la pérdida. Paidós ed. Barcelona: 2002.

3. Corral M, Matellanes F, Pérez J. El impacto psicológico del cáncer de pulmón en el paciente y su familia. Mapfre Med 2007;18 (2):108-13.

4. Gómez B, Novellas A, Alburqueque E, Schroder M. Información y comunicación en enfermos con cáncer avanzado y terminal. JANO 203; LXV(1495):56-64.

5. Stajduhar Kl, Thorne SE, McGuinness L, Kim-Sing C. Patient perceptions of helpful communication in the context of advanced cancer. J Clin Nurs 2010; 19(13-14):2039-47.
6. Navarro.E, Limonero J, Maté J. Necesidades de comunicación e información en el paciente oncológico superviviente. Psicooncología 2010;7(1):127-41.

7. Hack TF, Degner LF, Parker PA. The communication goals and needs of cancer patients: a review. Psychooncology 2005;14(10):831-45. Doi: 10.1002/pon.949.

8. Vogel BA, Leonhart R, Helmes AW. Communication matters: the impact of communication and participation in decision making on breast cancer patients' depression and quality of life. Patient Educ Couns 2009;77(3):391-7. Doi: 10.1016/j. pec.2009.09.005.

9. Black K, Emmet C. Nurses' advance care planning communication: an investigation. Geriatr Nurs 2006; 27 (4):222-7. Doi: 10.1016/j.gerinurse.2006.03.004.

10. Friedrichsen MJ, Strang PM, Carlsson ME. Receiving bad news: experiences of family members. J Palliat Care 2001;17(4):241-7. Doi: 10.1191/0269216302pm543oa.

11. Friedrichsen MJ, Strang PM, Carlsson ME. Cancer patients' interpretations of verbal expressions when given information about ending cancer treatment. Palliat Med 2002;16(4):323-30. Doi: 10.1191/0269216302pm543oa.

12. Morita $T$, Akechi $T$, Ikenaga $M$, Kizawa $Y$, Kohara H, MukaiyamaT, etal.Communication about the ending of anticancer treatment and transition to palliative care. Ann Oncol 2004;15(10):1551-7. Doi:10.1093/annonc/ mdh386.

13. Baile W, Buckman R, Lenzi R, Glober G, Beale EKA. Spikes- A six-step protocol for delivering bad news: application to the patient with cancer. Oncologist 2000; (5):302-11.

14. Serdà B. Evaluación de los efectos de un programa de ejercicio físico individualizado sobre la calidad de vida, la incontinencia urinaria, la fatiga y la resistencia muscular con enfermos de cáncer de próstata. Universitat de Girona; 2009. 
15. Serdà B, delValle A, Marcos R, Monreal P. Beneficios de un programa de ejercicio de fuerza para la mejora de la calidad de vida del hombre con cáncer de próstata. Psicooncología 2009;6(1):211-26.

16. Ospina A. El paciente terminal y la familia, el proceso de duelo y la acción profesional. Cali: Universidad del Valle 2002.

17. Kubler-Ross E. ["Pain and suffering of the dying"--a lecture. With love and honesty]. Krankenpfl Soins Infirm 1992; 85 (6):57-61.

18. Johansson M, Ryden A, Finizia C. Self evaluation of communication experiences after laryngeal cancer - a longitudinal questionnaire study in patients with laryngeal cancer. BMC Cancer 2008;8:80. Doi: 10.1186/1471-2407-8-80.

19. Kruijver IP, Kerkstra A, Francke AL, Bensing JM, van de Wiel HB. Evaluation of communication training programs in nursing care: A review of the literature. Patient Educ Couns 2000;39:121-45. Doi: 10.1016/S0738-3991(99)00096-8.

20. Rubin B, Blonch E. Intervención en crisis y respuesta al trauma: Teoría y práctica. Desclée de Brower ed. Bilbao: 2001.

21. Worden W. El tratamiento del duelo: asesoramiento psicológico y terapia. Paidos ed. Barcelona: 1997.

22. Clavero P, Cunill M. Rituales de despedida y conmemoración: la celebración de una vida. Sufunción preventiva en el proceso de duelo. Girona: Alfinlibros, 2008.

23. Denzin N, Lincoln Y. Handbook of qualitative research (2nd ed.). Thousand Oaks ed. CA: 2000.

24. Taylor SJ, Bogdan R. A qualitative approach to the study of community adjustment. Monogr Am Assoc Ment Defic 1981;(4):71-81.
25. Norman A, Sisler J, Hack T, Harlos M. Family physicians and cancer care. Palliative care patients' perspectives. Can Fam Physician 2001; 47: 2009-6.

26. Lucille AJ. [Teaching nursing homes in the United States]. Zhonghua Hu Li Za Zhi 1989;24 (9):515-7.

27. Morita $T$, Akechi $T$, Ikenaga $M$, Kizawa $\mathrm{Y}$, Kohara $\mathrm{H}$, Mukaiyama $\mathrm{T}$, et al. Communication about the ending of anticancer treatment and transition to palliative care. Ann Oncol 2004;15 (10):1551-7. Doi: 10.1093/annonc/ mdh386.

28. Mystakidou K, Tsilika E, Parpa E, Athanasouli P, Galanos A, Anna P, et al. Illness-related hopelessness in advanced cancer: influence of anxiety, depression, and preparatory grief. Arch Psychiatr Nurs 2009;23 (2):138-47. Doi: 10.1016/j. apnu.2008.04.008.

29. Eliott JA, Olver IN. Hope, life, and death: a qualitative analysis of dying cancer patients' talk about hope. Death Stud 2009;33 (7): 609-38. Doi: 10.1080/07481180903011982.

30. Munday D, Petrova M, Dale J. Exploring preferences for place of death with terminally ill patients: qualitative study of experiences of general practitioners and community nurses in England. BMJ 2009; 339:b2391. Doi: 10.1136/bmj.b2391.

31. Maté J, Mateo D, Bayés R, Bernaus $M$, Casas C, González-Barboteo J, et al. Elaboración y propuesta de un instrumento para la detección de malestar emocional en enfermos al final de la vida. Psicooncología 2009;6 (2-3):507-18. 
